\title{
Tunable Radiation Source by Coupling Laser-Plasma-Generated Electrons to a Periodic Structure
}

\author{
Z. Jin (金展), ${ }^{1}$ Z. L. Chen (陈正林), ${ }^{3}$ H. B. Zhuo (卓红斌), ${ }^{3,5}$ A. Kon (今亮), ${ }^{3}$ M. Nakatsutsumi (中堤基彰), ${ }^{3}$ \\ H. B. Wang (王红斌), ${ }^{2}$ B. H. Zhang (张保汉), ${ }^{2}$ Y. Q. Gu (谷渝秋), ${ }^{2}$ Y. C. Wu (吴玉迟), ${ }^{2}$ B. Zhu (朱斌), ${ }^{2}$ \\ L. Wang (王砧), ${ }^{2}$ M. Y. Yu (郁明阳), ${ }^{6,7}$ Z. M. Sheng (盛政明), ${ }^{8,9}$ and R. Kodama (兒玉了祐) ${ }^{1,3,4}$ \\ ${ }^{1}$ Photon Pioneers Center, Osaka University, 2-1 Yamada-oka, Suita, Osaka, 565-0871 Japan \\ ${ }^{2}$ National Key Laboratory of Laser Fusion, CAEP, Mianyang 621900, People's Republic of China \\ ${ }^{3}$ Graduate School of Engineering, Osaka University, 2-1 Yamada-oka, Suita, Osaka, 565-0871 Japan \\ ${ }^{4}$ Institute of Laser Engineering, Osaka University, 2-6 Yamada-oka, Suita, Osaka, 565-0871 Japan \\ ${ }^{5}$ School of Science, National University of Defense Technology, Changsha 410073, People's Republic of China \\ ${ }^{6}$ Institute for Fusion Theory and Simulation, Zhejiang University, Hangzhou 310027, People's Republic of China \\ ${ }^{7}$ Institute for Theoretical Physics I, Ruhr University, Bochum D-44780, Germany \\ ${ }^{8}$ Key Laboratory for Laser Plasmas (Ministry of Education) and Department of Physics, Shanghai Jiao Tong University, \\ Shanghai 200240, People's Republic of China \\ ${ }^{9}$ Beijing National Laboratory for Condensed Matter Physics, Institute of Physics, CAS, Beijing 100080, People's Republic of China
} (Received 9 June 2011; published 22 December 2011)

\begin{abstract}
Near-infrared radiation around $1000 \mathrm{~nm}$ generated from the interaction of a high-density MeV electron beam, obtained by impinging an intense ultrashort laser pulse on a solid target, with a metal grating is observed experimentally. Theoretical modeling and particle-in-cell simulation suggest that the radiation is caused by the Smith-Purcell mechanism. The results here indicate that tunable terahertz radiation with tens $\mathrm{GV} / \mathrm{m}$ field strength can be achieved by using appropriate grating parameters.
\end{abstract}

DOI: 10.1103/PhysRevLett.107.265003

PACS numbers: 52.25.Os, 52.59.Ye

Coherent, tunable sources of infrared (IR) and terahertz $(\mathrm{THz})$ radiation are of interest to many areas of research. Such light sources have many modern applications, such as for characterizing semiconductors and high-temperature superconductors, THz imaging of biomedical tissues, cellular structures, and dielectric substances, manipulation of bound atoms, etc. It is well known that strong $\mathrm{THz}$ radiation can be produced directly by the interaction of ultrashort intense laser pulses with gas targets [1]. It is also proposed that strong radiation sources ranging from $\mathrm{THz}$ to $\mathrm{x}$ ray can be obtained by ultrashort electron bunches either from conventional accelerators or laser-plasma acceleration [2,3]. Leemans et al. [2] obtained intense longwavelength radiation from femtosecond electron-bunch interaction with the plasma-vacuum boundary. In most schemes for generating IR and $\mathrm{THz}$ radiation, subpicosecond several-nC electron bunches are needed. Petawatt-laser interaction with solid targets can create $\mathrm{MeV}$ electron pulses with current densities of $10^{12} \mathrm{~A} / \mathrm{cm}^{2}$ [4]. However, in applications it is difficult to realize high peak-power coherent radiation because divergence of the electron bunches can rapidly reduce the current density to $10^{6} \mathrm{~A} / \mathrm{cm}^{2}$ or less within a short distance from the source.

In this Letter, we report experimental, theoretical, and numerical investigations on the generation of intense radiation between near-infrared (NIR) and far-infrared (FIR) by energetic electron beams from the interaction of intense lasers with solid targets. A tailored wedge target is used for obtaining the high-density electron beams as well as for self-consistently guiding and collimating the latter as they propagate along its grated surface. In the experiment, NIR radiation around $1000 \mathrm{~nm}$ is observed. The emission is attributed to Smith-Purcell radiation (SPR) arising from the coupling of the high-density relativistic beam electrons with the grating structure at the wedge surface [5].

The experiments were performed at the SILEX-I laser facility of the National Key Laboratory of Laser Fusion, Chinese Academy of Engineering Physics, and the P3 laser facility of the Graduate School of Engineering, Osaka University. The SILEX-I outputs $3 \mathrm{~J}, 800 \mathrm{~nm}, 30 \mathrm{fs}$ pulses focused into a $5 \mu \mathrm{m}$ spot by an $f 2,5$ off-axis parabolic mirror, resulting in a peak laser intensity of $5 \times 10^{20} \mathrm{~W} / \mathrm{cm}^{2}$. The experimental configuration is shown in Fig. 1(a). A $1 / 830 \mathrm{~mm}$ grating is chosen to realize output radiation in the NIR range, to which our spectrometer and CCD camera are sensitive. For comparison, another target with the same specifications but having a smooth flat upside surface is also used. Both targets are made of BK7 glass, with the upside surface coated with gold. The radiation emitted from the upside surface of the target due to the laser-plasma interaction on the target surface is collected simultaneously by a CCD camera and a spectrometer in the imaging system, as shown in Fig. 1(a). A knife edge is used to block the stray light from the laser spot region. That light is usually quite strong and could affect the radiation measurement. A bandpass filter is used to filter off the $<900 \mathrm{~nm}$ radiation. To assure that no 

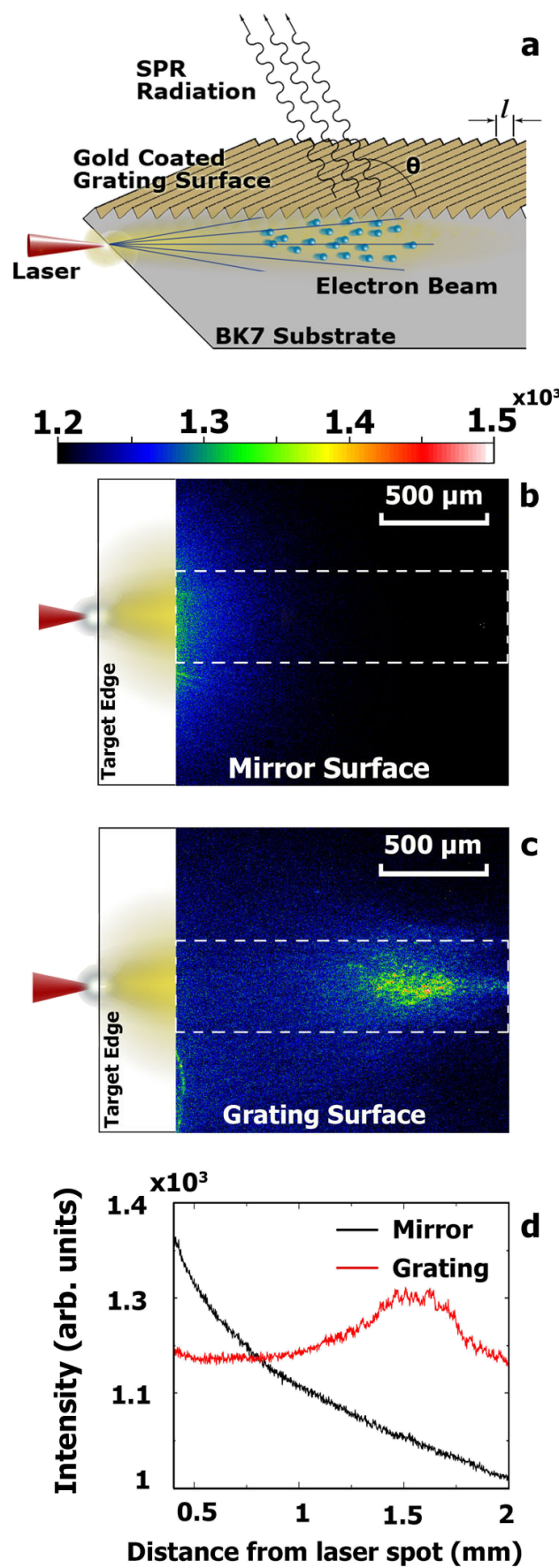

FIG. 1 (color online). (a) Experimental configuration of the electron beam and the grating structure. Images at the emission, or upside, surface of the solid target for targets with smooth (b) and grated (c) surfaces, as recorded by the CCD camera with a spatial resolution of $8 \mu \mathrm{m}$. The color bar shows the radiation intensity. The target front surface (solid line) for the laser irradiation (red cone) is on the left side. The laser-plasma interaction at the front surface and the resulting energetic electron beam are schematically shown. A knife edge blocks the scattered light originating from the focal spot region. The spatial distribution (d) of the emission intensity along the laser and electron propagation directions is obtained by integrating the radiation along the transverse direction in the region enclosed by the white dashed lines in (b) and (c). scattered light can arrive at the CCD camera and the spectrometer, the entire imaging system is enveloped and sealed in a black box.

The spatial distributions of the NIR radiation from the targets with grated and smooth upside surfaces are shown in Figs. 1(b)-1(d). The effect of the grating on the radiation can be clearly seen by comparing the radiation patterns from the grated and smooth surfaces in Figs. 1(b) and 1(c), respectively. A bright radiating spot, of width $500 \mu \mathrm{m}$ and nearly flat intensity distribution, on the grated surface is recorded by the CCD camera. In contrast, with the smooth surface the emission intensity reaches a peak close to the spot where the laser hits the target and decreases exponentially away from it. The energetic electron beam originating from the laser spot, which is $115 \mu \mathrm{m}$ below the grated surface, first propagates inside target plasma, and SPR is emitted when it reaches and propagates along the grated surface. The emission intensity distribution shown in Fig. 1(d) indicates a divergence angle of $10^{\circ}-25^{\circ}$ for those energetic electrons $(>10 \mathrm{MeV})$ contributing to SPR generation. This is consistent with that (typically $10^{\circ}-30^{\circ}$ ) of laser-accelerated hot electrons in overdense plasmas and can be attributed to the confinement effect of the selfgenerated magnetic fields [6,7].

For the analytical modeling, the laser-accelerated electron beam is assumed to be a low-emittance Gaussian electron bunch with temperature $T_{e}$ and length $\sigma$ propagating stably over the grating. The total angular spectral density of the radiation from the bunch may be written as [8]

$$
\begin{aligned}
\frac{d P_{n}}{d \Omega}= & \frac{N_{g} e n^{2}}{2 l \varepsilon_{0}} \frac{\beta^{3} I \sin ^{2} \theta}{(1-\beta \cos \theta)^{3}}\left[1+N_{e} f(\sigma, \theta)\right] \\
& \times \exp \left[\frac{-4 \pi|n| b}{l \gamma(1-\beta \cos \theta)}\right]\left|R_{n}\right|^{2},
\end{aligned}
$$

where $N_{e}$ is the bunch population, $N_{g}$ the period number of the grating, $I$ the beam current, $-e$ the electron charge, $\varepsilon_{0}$ the vacuum permittivity, $b$ the distance between the beam and the grating surface, $l$ the grating period, $\theta$ the observation angle, $n$ the harmonic order of the radiation, $\beta=v / c$ the normalized electron velocity, $c$ the light speed, $\gamma=$ $\left(1-\beta^{2}\right)^{-1 / 2}$ the electron relativistic factor, $\left|R_{n}\right|^{2}$ is associated with the efficiency of the grating structure, and $f(\sigma, \theta)=\exp \left[-(\pi \sigma \cos \theta / \lambda)^{2}\right]$ describes the coherence effect associated with the electron bunch's profile [9]. If the bunch length $\sigma$ is much smaller than the emission wavelength $\lambda$, one obtains intense coherent emission. Otherwise, only weak incoherent emission is produced. The corresponding wavelength $\lambda$ of the radiation of a single electron is given by [10]

$$
\lambda=(1-\beta \cos \theta) l / \beta|n| .
$$

In the experiment, the length $\sigma$ of the electron bunch is about $18 \mu \mathrm{m}$ after $1.5 \mathrm{~mm}$ of propagation, and the electron 
temperature as measured is about $8 \mathrm{MeV}$. Figure 2(a) shows the energy dependence of the normalized radiation efficiency for a single electron and the electron bunch, as given by Eq. (1). For the single electron, the radiation intensity (solid black line) increases (nonlinearly) with the electron energy. However for the electron bunch with a temperature of $8 \mathrm{MeV}$, the total radiation intensity (solid red line) peaks at $20 \mathrm{MeV}$ and then drops rapidly because of a corresponding decrease of the electron number. Over $90 \%$ of the radiation power can be attributed to the hot $(>10 \mathrm{MeV})$ electrons in the gray-shaded area. The blue curve in Fig. 2(a) shows the dependence of the radiation wavelength on the electron energy at $79.7^{\circ}$ observation angle. According to Eq. (2), at any given observation angle the radiation wavelength of these electrons should be almost the same. Figure 2(b) shows the radiation spectra measured by the spectrometer. Two peaks of the radiation
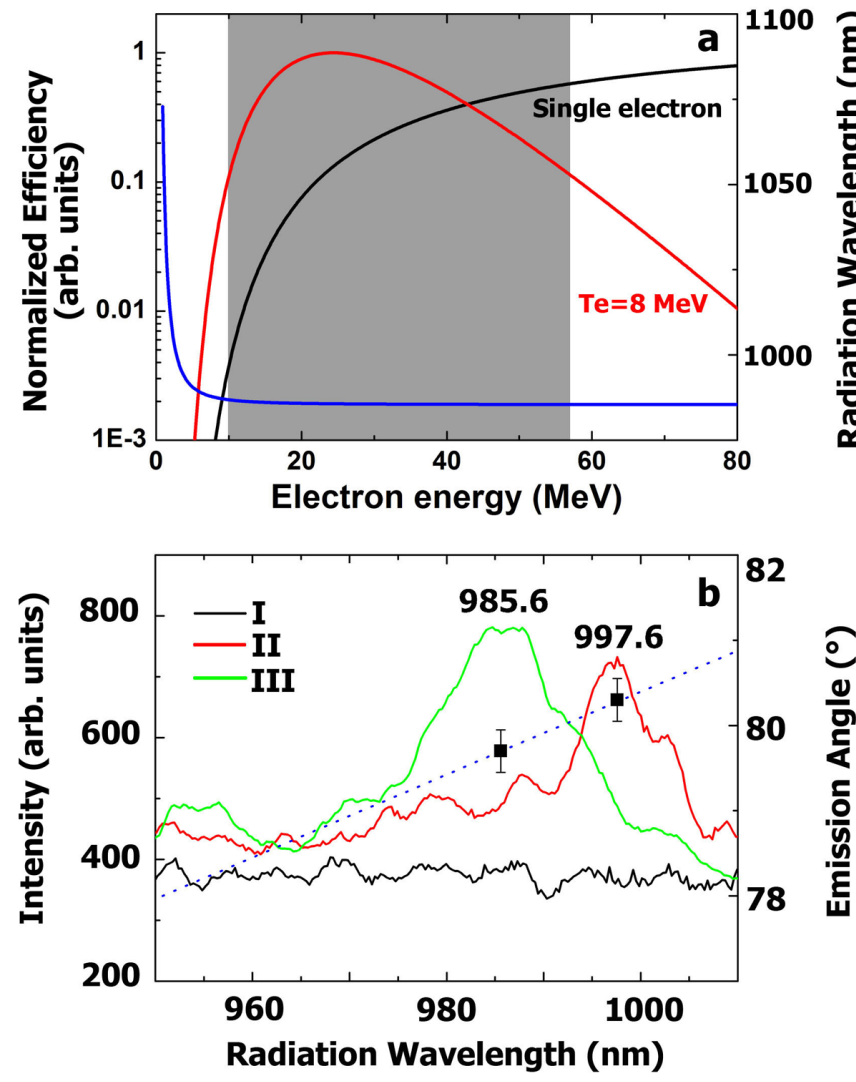

FIG. 2 (color online). (a) Dependence of the normalized SPR efficiency on the electron energy for a single electron and an electron bunch with $T_{e}=8 \mathrm{MeV}$ are shown by the black and the red solid curves, respectively. The dependence of the radiation wavelength on the electron energy at an observation angle $\theta=79.7^{\circ}$ is given by the blue line. (b) The black (I), the red (II), and the green (III) solid lines show the experimental radiation spectra from the smooth surface and the grated surface at $\theta=80.3^{\circ}$ and from the grating surface at $\theta=79.7^{\circ}$, respectively. The black dots show the calculated relation between the emission angle and the wavelength, and the black squares are the corresponding experimental results. emitted from the grating surface with quasisingle frequency are observed. The spectrum peak (black squares), shifting from 985.6 to $997.6 \mathrm{~nm}$ as the observation angle changes from $79.7^{\circ}$ to $80.3^{\circ}$, agrees perfectly with the theoretical result (dashed line) from Eq. (1). For the smooth-surface target, only very weak emission in this frequency range can be detected. The weak emission can be attributed to other mechanisms such as transition [11] and blackbody [12] radiation at low efficiencies.

To further verify the radiation mechanism, 2D particlein-cell (PIC) simulations are carried out using the PDLPICC2D code [13]. In the simulations, the wedge target is assumed to be a fully ionized plasma of initial density $n=100 n_{c}$, where $n_{c}$ is the critical density. Its upper surface has a sawtooth grating structure. The height of the sawtooth is $2 \mu \mathrm{m}$, and spatial periods from 5 to $20 \mu \mathrm{m}$ are considered. A linearly $p$-polarized laser, Gaussian in the transverse and axial directions, is focused on the wedge target $12.5 \mu \mathrm{m}$ below the grating surface with a spot radius of $5 \mu \mathrm{m}$. The laser intensity is $1.37 \times 10^{20} \mathrm{~W} / \mathrm{cm}^{2}$, the wavelength is $1 \mu \mathrm{m}$, and the pulse duration is $33 \mathrm{fs}$.

In all the cases simulated, we found that SPR is emitted as the hot electron bunch propagates along the grating surface. The hot-electron temperature $T_{e}$ is about $5 \mathrm{MeV}$, which is obtained from Wilks' scaling law [14]. As long as the electrons propagate stably, the low frequency radiation continues. Figure 3 illustrates the simulation results for two gratings: $l=10 \mu \mathrm{m}$ and $l=20 \mu \mathrm{m}$. The crescent-shaped wave fronts of the low frequency radiation shown in Figs. 3(a) and 3(c) correspond to those of the classical
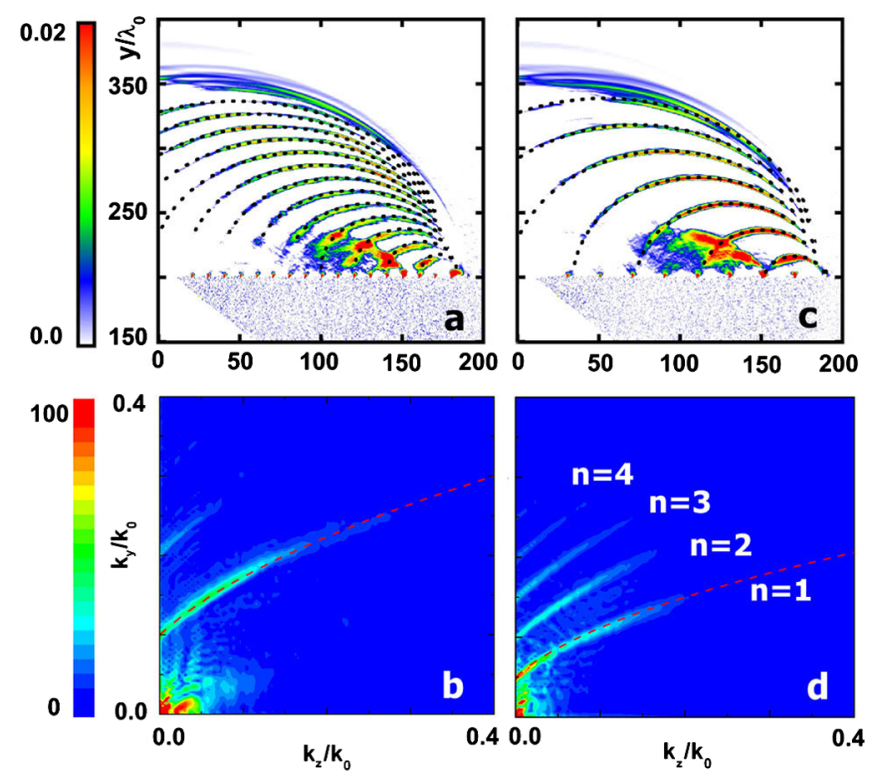

FIG. 3 (color online). Snapshots from the 2D PIC simulations. (a),(b) The emission field $e E_{z} / m c \omega_{0}$ and the Fourier spectrum $E_{z}(k)$ for $l=10 \mu \mathrm{m}$ and (c),(d) $l=20 \mu \mathrm{m}$ at 200 laser periods. The black dots and the red dashes represent the calculated first order SPR results. 
Smith-Purcell result [15]. The Fourier spectra of the emission fields for $l=10 \mu \mathrm{m}$ and $l=20 \mu \mathrm{m}$ are shown in Figs. 3(b) and 3(d), respectively. One can clearly see that for both cases the results (black dots and red dashed line) from the first order Smith-Purcell relation for nearlight-speed electrons agree well with those from the simulations. That is, the emission is indeed mainly from the very fast electrons. We also see that the grating period affects both the amplitude and the spectrum of the SPR emission. The maximum radiation amplitude for $l=$ $20 \mu \mathrm{m}$ is $e E_{z} / m \omega_{0} c \sim 0.03$ (corresponding to a field of $9 \times 10^{10} \mathrm{~V} / \mathrm{m}$ ), which is about 1.5 times that for $l=10 \mu \mathrm{m}$. For longer grating periods even higher order emissions can be obtained. In fact, harmonic emission up to the fourth order can be found in Fig. 3(d), although only the second harmonic emission is observable in Fig. 3(b).

Figures 4(a) and 4(b) show the transverse fields at the surface of the target. The radial electric field $E_{y}$, or the charge separation field induced by the escape of highdensity $\mathrm{MeV}$ electrons, acts to pull the slower electrons back to the surface. A strong negative azimuthal magnetic field $B_{x}$, of the order of a few hundred megagauss, is created and it tends to confine the electrons. Since the hot electrons have speeds close to that of light, balance of the electric $\left(e E_{y}\right)$ and magnetic $\left(e v_{e} B_{x} / c\right)$ forces tends to confine and collimate the electrons, so that the latter can propagate stably along the grated surface [16].

In view of the theoretical expression (1), the results here indicate that the NIR radiation found in our experiments can be tuned to the THz range by a straightforward scheme. For example, with the same experimental parameters, increasing the grating period $l$ to $1 \mathrm{~mm}$ would increase the radiated wavelength at $\theta=90^{\circ}$ to $1 \mathrm{~mm}$. Furthermore, Fig. 5 shows that when the grating period is larger than or comparable to the electron-bunch length, the emission intensity abruptly increases [because of the coherency factor $f(\sigma, \theta)$, which is represented by the green-blue curve]. It is found that the $1.2 \mu \mathrm{m}$ grating results in incoherent SPR emission, which is usually weak. We have also simulated this case, but the SPR emission is so weak that it is submersed in the background noise.

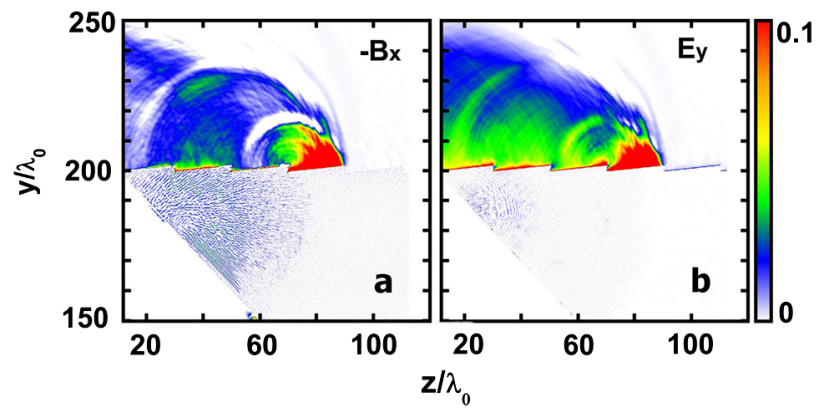

FIG. 4 (color online). (a) The transverse electric field $e E_{y} / m c \omega_{0}$ and (b) magnetic field $e B_{x} / m \omega_{0}$ for $l=20$ at 100 laser periods.
However, in the $\mathrm{THz}$ regime (gray-shaded area in Fig. 5) the coherent enhancement is about $8-10$ orders of magnitude higher than the incoherent case for the measured bunch electron number $10^{11}$. Deconvolution of the efficiency of the spectrometer shows that the power of the incoherent NIR radiation found in our experiments is $\sim 3.8 \times 10^{2} \mathrm{~W} / \mathrm{cm}^{2}$. For the same laser parameters, as the grating period is tuned to $20 \mu \mathrm{m}$, PIC simulation suggests a radiation intensity of $3 \times 10^{12} \mathrm{~W} / \mathrm{cm}^{2}$. In Fig. 5 the experimental and simulation normalized intensities are shown as black and gray (red) solid squares, respectively. The enhancement effect is consistent with the curve obtained from Eq. (1). Thus one should be able to realize a very powerful $\mathrm{THz}$ source by improving the electron beam quality [17] and/or optimizing the coupling between the electron bunch and the target.

In conclusion, for the first time NIR emission around $1000 \mathrm{~nm}$ has been experimentally realized by coupling a periodic grating surface to the energetic electron bunches generated in intense ultrashort pulse laser interaction with plasma. It is identified to be due to the Smith-Purcell effect. Since the electron-bunch length is larger than the grating period of about $1.2 \mu \mathrm{m}$ in the experiment, the emission is incoherent. On the other hand, the radiation wavelength can be controlled by varying the grating period and the observation angle. PIC simulation and theory indicate that the radiation becomes coherent as the grating period is increased to larger than $10 \mu \mathrm{m}$. As a result, its intensity can be enhanced by an order of magnitude, similar to the number of the $\mathrm{MeV}$ electrons. This scheme can thus be useful for realizing an intense tunable tabletop $\mathrm{THz}$ farinfrared FIR radiation source.

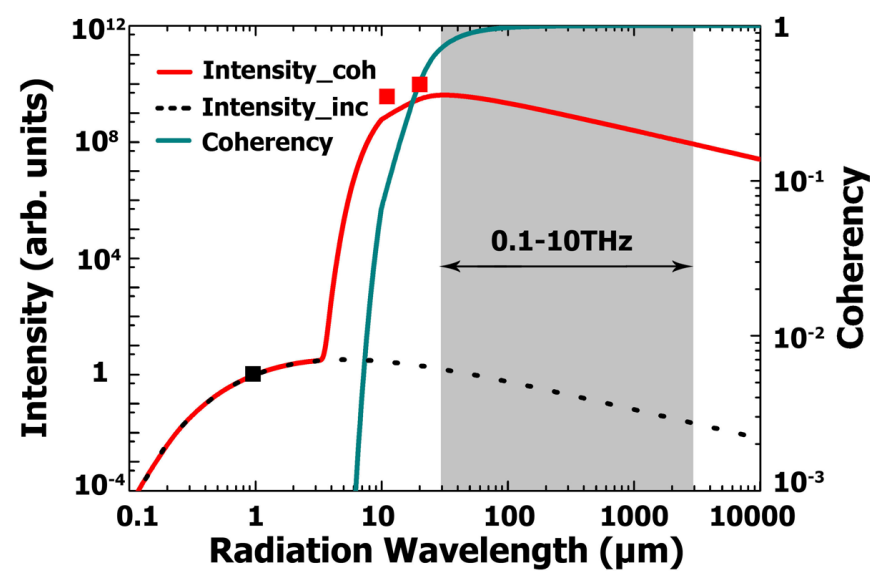

FIG. 5 (color online). Dependence of the radiation intensity on the wavelength with (red solid line) and without (black dotted line) the coherence factor. The latter depends on the radiation wavelength and is given by the blue-green solid curve. The radiation intensities measured from experiments and PIC simulations are shown as the black and gray (red) solid squares, respectively. The intensities are normalized by that of the incoherent $1 \mu \mathrm{m}$ radiation. 
This work is supported by the Core Research for Evolutional Science and Technology, Japan Science and Technology Agency. It is partially supported by the grants for the Global COE Program, "Center for Electronic Devices Innovation," from the Ministry of Education, Culture, Sports, Science and Technology of Japan. H. B. Z. and M. Y. Y. would like to acknowledge the support of the National Natural Science Foundation of China (Grants No. 10976031, No. 10975185, No. 10835003, and No. 11175253). Z.M.S. acknowledges the support of the National Basic Research Program of China (Grants No. 2007CB310406, No. 2007CB815100, and No. 2009GB105002).

[1] X.-C. Zhang et al., Appl. Phys. Lett. 56, 1011 (1990); R. Kersting et al., Phys. Rev. Lett. 79, 3038 (1997); P. Sprangle et al., Phys. Rev. E 69, 066415 (2004); Mark D. Thomson et al., Laser Photon. Rev. 1, 349 (2007); K. Reimann, Rep. Prog. Phys. 70, 1597 (2007); H.-C. Wu et al., Phys. Rev. E 83, 036407 (2011) and the references therein.
[2] W.P. Leemans et al., Phys. Rev. Lett. 91, 074802 (2003); G.L. Carr et al., Nature (London) 420, 153 (2002);

M. Abo-Bakr et al., Phys. Rev. Lett. 90, 094801 (2003).

[3] M. Fuchs et al., Nature Phys. 5, 826 (2009).

[4] J. Faure et al., Nature (London) 431, 541 (2004).

[5] G. Doucas et al., Phys. Rev. Lett. 69, 1761 (1992).

[6] P. A. Norreys et al., Plasma Phys. Controlled Fusion 48, L11 (2006).

[7] M. Borghesi et al., Phys. Rev. Lett. 83, 4309 (1999).

[8] K. J. Woods et al., Phys. Rev. Lett. 74, 3808 (1995).

[9] M. C. Lampel, Nucl. Instrum. Methods Phys. Res., Sect. A 385, 19 (1997).

[10] S. J. Smith and E. M. Purcell, Phys. Rev. 92, 1069 (1953).

[11] G. M. Garibian et al., Sov. Phys. JETP 6, 1079 (1958).

[12] M. Planck, The Theory of Heat Radiation (Blackiston's Son and Co., Philadelphia, PA, 1914), 2nd ed.

[13] H. B. Zhuo et al., Phys. Rev. Lett. 105, 065003 (2010).

[14] S. C. Wilks et al., Phys. Rev. Lett. 69, 1383 (1992).

[15] A. S. Kesar et al., Phys. Rev. E 71, 016501 (2005).

[16] Y. T. Li et al., Phys. Rev. Lett. 96, 165003 (2006).

[17] R. Kodama et al., Nature (London) 432, 1005 (2004). 